\title{
Non-reciprocal Interferometers for Matter Waves
}

\author{
J. Mannhart ${ }^{1}$ \\ Received: 21 December 2017 / Accepted: 6 March 2018 / Published online: 26 March 2018 \\ (C) The Author(s) 2018
}

\begin{abstract}
We propose non-reciprocal interferometers for matter waves and explore them by means of modeling. These interferometers may be implemented as asymmetric quantum rings with broken time-inversion symmetry. Our preliminary analyses lead us to predict that these devices will feature asymmetric transport properties for particles such as electrons, for which the interferometers' ground states act as directional filters. As a function of the electron velocity, the filters seem to let electrons preferentially pass in one direction rather than in the reverse, thereby reducing the entropy of the electron systems in the contacts. Mechanisms are discussed that are candidates to prevent directional filtering if the filters are operating in thermal equilibrium with incoherent electron reservoirs.
\end{abstract}

Keywords Dissipation-free current · Non-reciprocal device · Maxwell demon

C'est la dissymétrie qui crée le phénomène Pierre Curie

\section{Introduction}

The discovery of superconductivity [1] enabled the transport of an electric charge without dissipation. Sustained, constructive work to develop sophisticated superconducting materials has led to enormous advances in the performance of superconductors and in raising their critical temperature [2, 3]. Interestingly, dissipation-free charge flow exists also in nonsuperconducting systems. Atoms, molecules, atomic clusters, and mesoscopic conducting rings may carry such currents; see, e.g., [4-6].

Quantum-Hall systems [7] also transport current lossfree if biased in a quantum-Hall plateau [7, 8]. Loss-free currents have furthermore been found to flow along the edges of topological insulators; see, e.g., [9]. The flow of these currents benefits from topological protection against back-scattering by elastic scattering events that preserve time-reversal symmetry. Indeed, the use of topologically

\footnotetext{
J. Mannhart

office-mannhart@fkf.mpg.de

1 Max Planck Institute for Solid State Research, 70569 Stuttgart, Germany
}

protected surface currents in chip interconnects has been proposed [10]. In all systems described, dissipation-free transport is based on sustained quantum coherence and the suppression of inelastic scattering.

One may also want to consider ratchets and Brownian motors [11, 12] to transport charge along channels or wires. Such ratchets are microscopic, time-reversalinvariant machines that move particles due to perturbations that drive the system. Fueled by operation out of equilibrium, ratchets are therefore not a suitable means to design loss-free conductors.

Here, we report on our search for further possibilities to realize loss-free charge flow in non-superconducting devices or wires, and propose non-reciprocal filters for electrons as candidates. This search has been motivated by the fundamental question of whether such a device or wire is realizable in principle and by the implications its sheer existence might have $[11,13]$. We present several proposals for such devices to initiate a discussion of their properties and underlying principles and to trigger further developments of this novel approach.

\section{Fundamental Requirements for Achieving Loss-Free Transport}

We first recall a classic theorem by Bloch [14, 15], which states that the ground state of a (almost arbitrary) system of electrons can only carry a current if the time-reversal invariance of the Hamiltonian is explicitly broken, for example, 
by an external magnetic field. Breaking time-reversal invariance in phase-coherent systems either by a magnetic field or a current bias may easily lead to persistent currents in superconductors, quantum-Hall systems, and mesoscopic configurations smaller than the inelastic scattering length.

But even in systems that are intrinsically time-reversal invariant, current flow with no voltage gradient may occur by spontaneous or by external time-reversal symmetry breaking. This current flow is not a ground-state property, however. The dc-Josephson effect is one example. In that case, the left-right asymmetry that determines the current direction is not caused by the barrier itself, which may be engineered to be perfectly symmetric, but by the phase difference of the superconducting condensate on both sides of the junction.

We consider here a possible current that is observable without applied voltage in normal-conducting devices in which time-reversal symmetry is broken by an applied magnetic field. In addition, the devices break spatialinversion symmetry. For simplicity, the systems are assumed here to be fully elastic such that no energy is dissipated in the device during particle transport. If the device is characterized as a scattering center in a uniform environment, the amplitudes $f\left(\boldsymbol{p}, \boldsymbol{s} ; \boldsymbol{p}^{\prime}, \boldsymbol{s}^{\prime}\right)$ for scattering across the device from state $(\boldsymbol{p}, \boldsymbol{s})$, where $\boldsymbol{p}$ is the momentum and $\boldsymbol{s}$ the spin, into state $\left(\boldsymbol{p}^{\prime}, \boldsymbol{s}^{\prime}\right)$, do not necessarily fulfill the relation

$\left|f\left(\boldsymbol{p}, \boldsymbol{s} ; \boldsymbol{p}^{\prime}, \boldsymbol{s}^{\prime}\right)\right|=\left|f\left(-\boldsymbol{p}^{\prime},-\boldsymbol{s}^{\prime} ;-\boldsymbol{p},-\boldsymbol{s}\right)\right|$.

Equation (1) follows from the reciprocity theorem for timereversal invariant scattering potentials [16]. We therefore suggest searching for devices for which the probability of an electron traversing the barrier from left to right is different from the probability of the time-reversed process, i.e.,

$\sum_{\boldsymbol{s}, \boldsymbol{s}^{\prime}}\left|f\left(\boldsymbol{p}, \boldsymbol{s} ; \boldsymbol{p}^{\prime}, \boldsymbol{s}^{\prime}\right)\right|^{2}>\sum_{\boldsymbol{s}, \boldsymbol{s}^{\prime}}\left|f\left(-\boldsymbol{p}^{\prime}, \boldsymbol{s}^{\prime} ;-\boldsymbol{p}, \boldsymbol{s}\right)\right|^{2}$

$[17,18]$. The device function we seek requires asymmetric transport between two equivalent contacts. Therefore, it cannot be obtained from conventional asymmetric bipolar electronic devices such as $p n$-diodes. Weak non-reciprocal resistance has been found in materials with magnetochiral anisotropy such as carbon nanotubes, where the effect is associated with a sizable sample resistance [19], and also in non-centrosymmetric superconductors [20]. We are searching for non-superconducting devices, however, that like a unidirectional membrane let particles pass or not pass in a non-reciprocal manner. Photonic non-reciprocal devices $[21,22]$ are devices that bear some analogies to the ones we have in mind.

\section{Proposals for Devices}

We seek devices wherein electrons would be transmitted from the left to the right with a higher probability than electrons approaching the barrier from the right. This also applies to electrons driven by thermal excitation (Fig. 1). To break the time-reversal symmetry, we focus on devices subject to a magnetic field, considering effects caused by the magnetic field that break the parity of the device in current-flow direction.

\subsection{Devices Using Phase Shifts Induced by the Rashba Effect}

The Rashba effect couples longitudinally to the particle's phase [23, 24]. In numerous studies, spin-orbit coupling and the related Rashba effect have indeed been suggested, explored, and sometimes even doubted as possible drivers for lossless currents in homogeneous materials and in heterostructures; see, e.g., [25-30].

The Rashba effect contributes kinetic energy to particles that carry a magnetic moment $\boldsymbol{\mu}$ and move in a transverse electric field $\boldsymbol{E}$. If a magnetic field $\boldsymbol{B}_{\mathbf{a}}$ also exists, the Hamiltonian of the electron is

$H=\hbar^{2} k^{2} / 2 m-\hbar k / m(\boldsymbol{\alpha} \times \boldsymbol{\sigma}) \cdot \hat{z}-\boldsymbol{\mu} \boldsymbol{B}_{\mathbf{a}}$.

Here, $k$ describes the wave number of the electron, where the canonical momentum is $\boldsymbol{p}=\hbar \boldsymbol{k}, m$ the electron mass, $\sigma$ the Pauli matrices, and the unit vector of the direction in which the electric field is applied. The Rashba momentum is $\boldsymbol{\alpha}=\hat{\alpha \boldsymbol{p}}$ with $\alpha=\mu E_{z} / 2 c^{2}$ where $E_{z}$ is the $z$-component of the electric field and $c$ the speed of light.

$$
T_{\text {con }} \quad T_{b} \quad T_{c o n}
$$
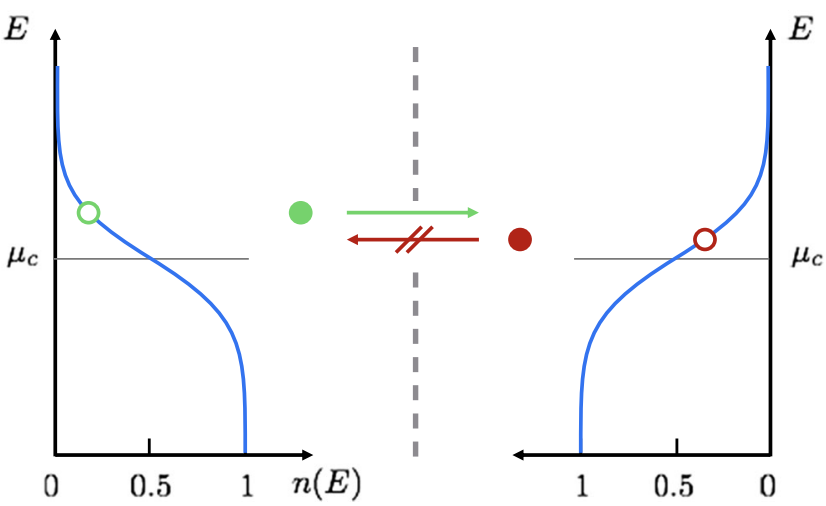

Fig. 1 Illustration of a hypothetical barrier that has a higher transparency for thermally excited electrons moving from the left metal to the right than for the ones moving from the right metal to the left. The parameters $E, \mu_{\mathrm{c}}$, and $n(E)$ denote the electron energy, the chemical potential, and the filled density of states, respectively. The temperature of the metals is $T_{\text {con }}$ and the barrier temperature is $T_{\mathrm{b}}$ 
For these devices, we now consider the case where $E_{z}=0$ and $\boldsymbol{B}_{\mathbf{a}}$ is oriented in the $y$-direction. The electrons move in the $x$-direction in an $x y$-oriented, two-dimensional quantum well of length $l$, and their motion is characterized by a parabolic dispersion $E=p^{2} / 2 m=\hbar^{2} k^{2} / 2 m$ The field $\boldsymbol{B}_{\mathbf{a}}$ is assumed to be so large that the Zeeman energy $E=-\boldsymbol{\mu} \boldsymbol{B}_{\mathrm{a}}$ is by far the predominant energy term, yielding virtually complete spin polarization. For this scenario, (3) simplifies to

$H=\hbar^{2} k^{2} / 2 m-\alpha \hbar k / m-\mu B_{\mathrm{a}}$,

where $k$ is now oriented in the $x$-direction. In this case, the dispersion parabola (Fig. 2a) is spin-split into two parabolas, as shown by Fig. $2 b$.

According to (4), these two parabolas are shifted in opposite directions along the $k$-axis when $\left|E_{z}\right|>0$. The absolute value of the shift is $\hbar k_{R}=\alpha$. As the chemical potential $\mu_{\mathrm{c}} \ll \mu B_{\mathrm{a}}$ the occupancy of the parabola with the high-energy spin direction is exponentially small. To simplify the problem, we therefore disregard this parabola also for $\left|E_{z}\right|>0$.

Striving to combine Rashba and non-Rashba materials in one device, we start by considering trilayer heterostructures. Figure 3 shows an example of heterostructures, in which a barrier layer affected by the Rashba effect connects two standard non-Rashba metals. If the canonical momentum of the electrons were conserved in these devices, which, however, is not the case, the devices would be good candidates for asymmetric transport. To illustrate this point, Fig. 3a sketches the transport across such a hypothetical device. In this case, the barrier layer would feature different effective barrier heights for electrons tunneling in opposite directions. However, when the electrons pass from a standard material into one with Rashba coupling, their canonical momentum $p=\hbar k$ increases by $\alpha$ independently of whether the electron travels from left to right or vice versa, Fig. 3b. The canonical momentum of the entire system is preserved because the electrons exchange the momentum with the electromagnetic field. Therefore, these devices do not feature asymmetric transport properties.

It is inspiring to note that, although the particle's kinetic energy $E=1 / 2 m v^{2}$ is symmetric in $v$ also for a finite Rashba momentum $\alpha$, the particle's canonical momentum and wave number are not symmetric: $p=\hbar k=m v+$ $\alpha$. Particles traveling with the same absolute velocity in opposite directions $v_{1}=-v_{2}$ are characterized by wave numbers with different absolute values

$\hbar k_{1}=m v_{1}+\alpha$

$\hbar k_{2}=m v_{2}+\alpha=-m v_{1}+\alpha$

see Fig. 4. The asymmetric relationship between $\hbar k$ and $v$ has been observed in numerous experiments; see, e.g., [31].

The problem of achieving asymmetric transport has now been mapped onto the more conventional problem of converting changes of the electron wavelength into changes of device transmission. As the electron phase is not gaugeinvariant, the electron phase in any such device must be compared with a reference phase. Therefore, we now consider utilizing waves $\Psi_{\mathrm{I}}(x, t)$ and $\Psi_{\mathrm{II}}(x, t)$ that pass a device on a Rashba path I and on a non-Rashba reference path II, respectively, and then interfere with each other. The layout of these devices is shown in Fig. 5. We note that related mesoscopic rings with inhomogeneous spin-orbit coupling have already been explored in a different context $[32,33]$. The two paths I and II may be compared to the two wave paths used in Aharonov-Bohm-type devices [34]. In our proposed device, path I induces a direction-dependent (a)

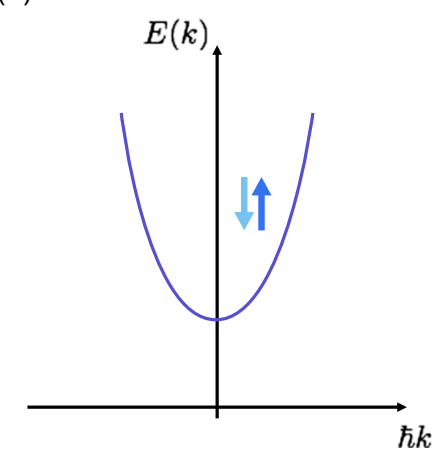

(b)

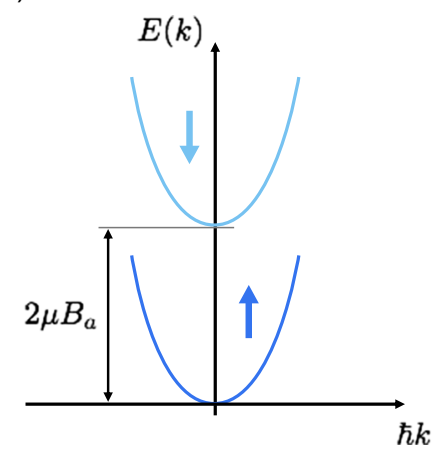

(c)

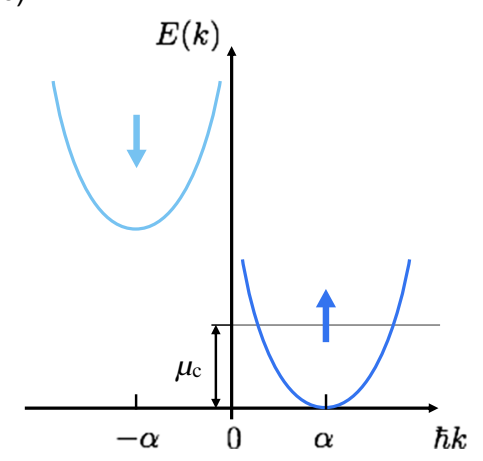

Fig. 2 Influence of the applied magnetic field $B_{\mathrm{a}}$ and of the Rashba effect on the band structure of material I. Panel a shows the standard parabolic dispersion of the material for $B_{\mathbf{a}}=0$ and $E_{\mathbf{a}}=0$ where $k$ is the wave number in the $x$-direction. Panel $\mathbf{b}$ illustrates the case where a large magnetic field $B_{\mathbf{a}}$ is applied and where $E_{\mathbf{a}}=0$. The magnetic field causes a spin splitting of the dispersion, such that two bands offset by twice the Zeeman energy are obtained. Panel $\mathbf{c}$ illustrates the case where both $B_{\mathbf{a}}$ and $E_{\mathbf{a}}$ are applied. The Rashba effect induces a shift of the two parabolas along the $k$-axis, such that their minima are positioned at $\hbar k=\alpha$ and at $\hbar k=-\alpha$, respectively. The chemical potential $\mu_{\mathrm{c}}$ in the device is chosen such that $\mu_{\mathrm{c}} \ll \mu B_{\mathbf{a}}$ 
(a)

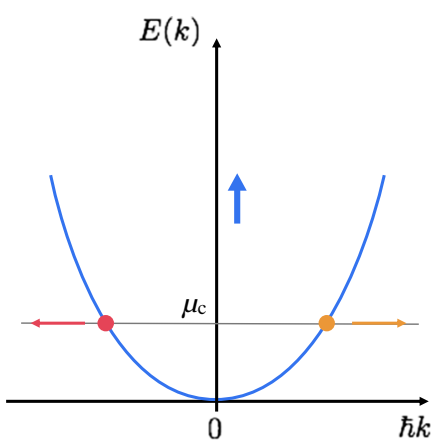

standard metal

(b)

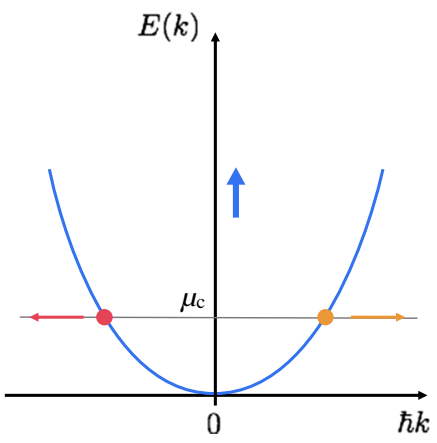

standard conductor

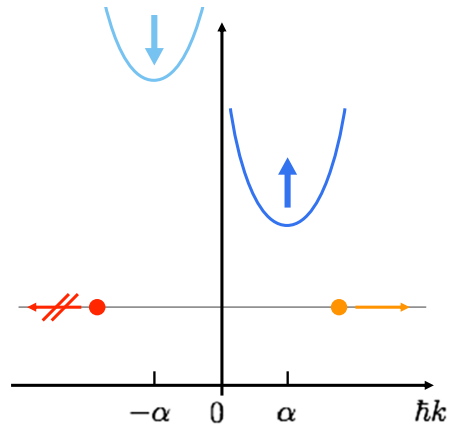

Rashba material

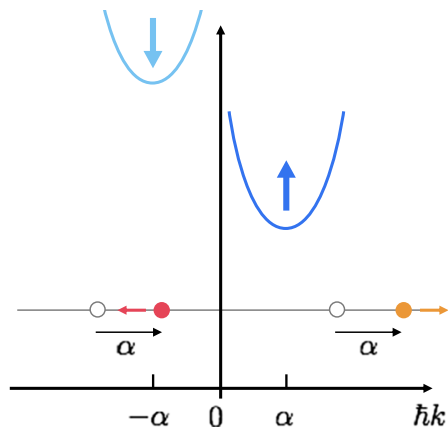

Rashba material

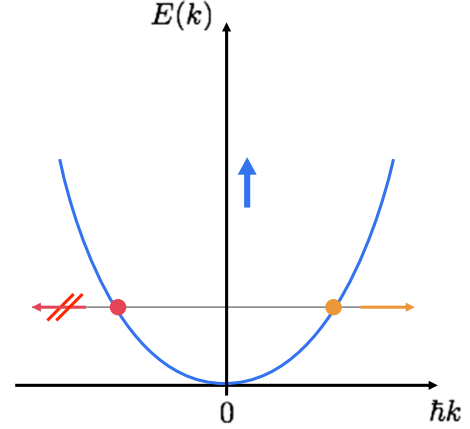

standard metal

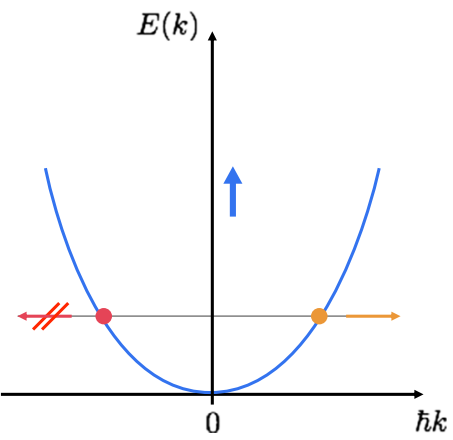

standard conductor
Fig. 3 Band structure of a tunnel junction with a barrier shaped by the Rashba effect. Panel (a) illustrates the case that such a device would show asymmetric transport if the canonical momenta of the electrons

shift $\delta \varphi_{\mathrm{I}, A B}(x) \neq \delta \varphi_{\mathrm{I}, B A}(x)$ of the wavefunctions' phase $\varphi(x)$, where $A$ and $B$ denote the two contacts of the device, and the index $A B$ specifies that the electron moves from $A$ to $B$. This phase shift is used to tune the particle's transmission probabilities $t_{A B}(v)$ and $t_{B A}(v)$ as the wave packet is made to interfere at the end of path I with the packet that travelled via path II. A particle entering the device at $A$ with $\left|\Psi_{A B}(A)\right|^{2}=1$ reaches terminal $B$ with a probability of $t_{A B}(v)=\left|\Psi_{A B}(B)\right|^{2}$. A particle entering the device at $B$ reaches $A$ with a probability of $t_{B A}(v)=$ $\left|\Psi_{B A}(A)\right|^{2}$.

To determine the phase change of the particle on path I, we first assess the phase change induced by the electron's total energy $\delta \varphi_{\mathrm{I}, A B, E}=E_{\mathrm{tot}} \Delta \tau$. Owing to $\left|v_{1}\right|=\left|v_{2}\right|$, the energy $E_{\text {tot }}=1 / 2 m v^{2}$ as well as the particle's travel time $\Delta \tau$ are independent of the travel direction $\delta \varphi_{\mathrm{I}, A B, E}=$ $\delta \varphi_{\mathrm{I}, B A, E}$. Note that the spin has been fixed. As $E_{\mathrm{tot}} \Delta \tau$ does not introduce a direction-induced phase change, it does not require further consideration.

With the assumption that the electron's phase changes can be described analogously to the phase changes of wave packets in conventional materials, (5) yield the phase change were conserved. As the electrons exchange momentum with the electromagnetic field when passing into and out of the barrier, symmetric transport is obtained, panel (b)

of an electron starting at $A(x=0)$ and traveling on path $\mathrm{I}$ to arrive at $B(x=l)$ as

$\Delta \varphi_{A B, \mathrm{I}}=\left(m v_{1}+\alpha\right) l$,

neglecting phase retardation at the beam splitters. An electron starting at $B$ and traveling to $A$ on path I changes its phase by

$\Delta \varphi_{B A, \mathrm{I}}=\left(m v_{1}-\alpha\right) l$.

Path II is designed such that there the Rashba effect vanishes, done, for example, by setting $\boldsymbol{E}=0$ for this path. Therefore

$\Delta \varphi_{A B, \mathrm{II}}=\Delta \varphi_{B A, \mathrm{II}}=m v_{\mathrm{II}} l$.

The length $l$ of the device is chosen such that the electron traveling on the two paths from $A$ to $B$ constructively interferes at $B: \Delta \varphi_{A B, \mathrm{I}}=\Delta \varphi_{A B, \mathrm{II}}+2 \pi n$, where $n$ is an integer: $l^{\prime}:=2 \pi n /(m \Delta v+\alpha)$, with $\Delta v:=v_{\mathrm{I}}-v_{\mathrm{II}}$. For $l=l^{\prime}$, the two parts of the waves traveling from $B$ to $A$ arrive at $A$ with a phase difference of

$\Delta \varphi_{B A, \mathrm{I}}-\Delta \varphi_{B A, \mathrm{II}}=\frac{m \Delta v-\alpha}{m \Delta v+\alpha} 2 \pi n$. 

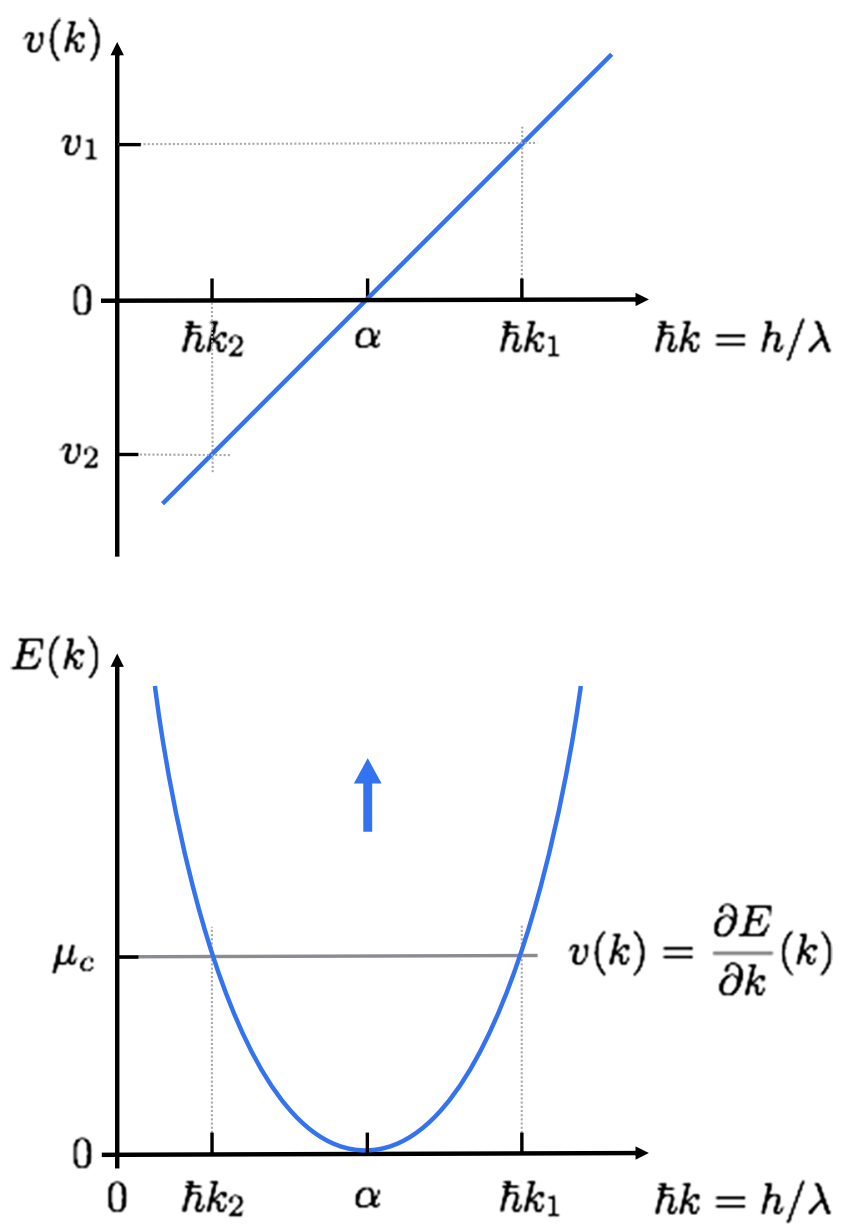

Fig. 4 Illustration of the energy dispersion and the dependence of the electron velocity $v$ on the canonical momentum in material I. Note how the two velocities $v_{1}$ and $v_{2}=-v_{1}$ correspond to two wave numbers and wavelengths $k_{1}, \lambda_{1}$ and $k_{2}, \lambda_{2}$, respectively, which have different absolute values

For $\Delta v \neq 0$ we find that $\Delta \varphi_{B A, \mathrm{I}}-\Delta \varphi_{B A, \mathrm{II}} \neq 2 \pi n$, except for the special case of $m \Delta v=\alpha$. For $\Delta \varphi_{B A, \mathrm{I}}-\Delta \varphi_{B A, \mathrm{II}} \neq$ $2 \pi n$, at least a part of the electron wave is reflected back to

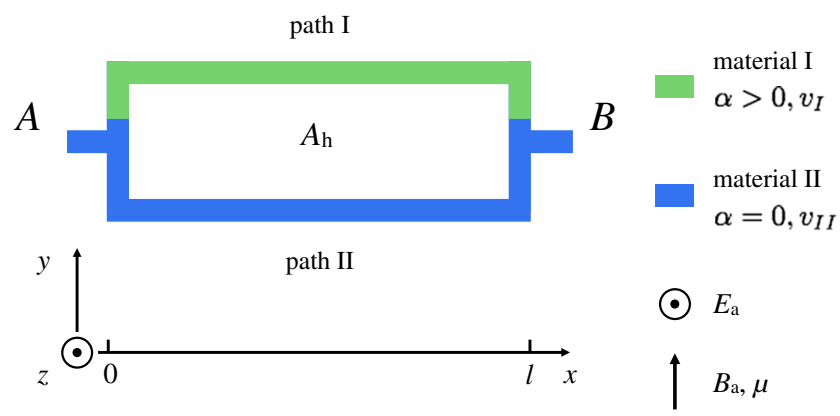

Fig. 5 Sketch of the device layout. The two materials I and II form a quantum ring of length $l$ that is contacted at its ends $A$ and $B$. An electric field $E_{\mathbf{a}}$ is applied in the $z$-direction. An applied magnetic field $B_{\text {a }}$ provides for a spin polarization such that the electron magnetic moments $\boldsymbol{\mu}$ point in the $y$-direction
$B$. On the path to $B$, this reflected wave acquires additional phase shifts which affect the probability for the particle to exit at $B$. Because this electron travels in the device a longer average path than the electron that entered at $A$, it is more likely to scatter inelastically, to then possibly leave the device via port $B$. We point out that the meanfree path for inelastic scattering of an electron of velocity $v$ must not be much smaller than $l^{\prime}$; see also $[35,36]$. The condition $\Delta v \neq 0$ can be fulfilled by selecting two different materials with appropriate band structures for paths I and II, as illustrated in Fig. 6. Therefore, our analysis shows that $t_{A B}(v) \neq t_{B A}(v)$ for devices for which (i) $l=l^{\prime}$, (ii) $m \Delta v \neq \alpha$, and (iii) $\Delta v \neq 0$.

The analysis entails that like a magnetic-field biased Aharonov-Bohm ring, a uniform, coherent loop that includes a Rashba conductor generates a circulating current in the ground state (see Fig. 7) with

$\frac{m}{q n_{q}} \oint \boldsymbol{j}(\boldsymbol{r}) d \boldsymbol{s}=n h-\oint(q \boldsymbol{A}(\boldsymbol{r})+\boldsymbol{\alpha}(\boldsymbol{r})) d \boldsymbol{s}$,

where $\boldsymbol{j}$ and $n_{q}$ denote the current density and the carrier density, respectively.

Before discussing possible effects that could jeopardize the functioning of the device, we will now analyze whether the Rashba effect is a prerequisite for directional transport.

\subsection{Devices Using Phase Shifts Induced by the Vector Potential}

To explore whether directional transport can be achieved without the Rashba effect, we express the Hamiltonian of (3) as

$H=1 / 2 m(m \boldsymbol{v}(\boldsymbol{r})+q \boldsymbol{A}(\boldsymbol{r})+\boldsymbol{\alpha}(\boldsymbol{r}))^{2}-\mu B$

Equation (10) makes it evident that $\boldsymbol{A}(\boldsymbol{r})$ and $\boldsymbol{\alpha}(\boldsymbol{r})$ coupling to the charge $q$ and to the magnetic moment $\mu$, respectively, have an equivalent influence on the electron phase. This equivalency suggests that devices with directional transport may also be realizable by replacing $\boldsymbol{\alpha}(\boldsymbol{r})$, which is not constant in the direction transverse to the motion, by a vector potential that is also oriented parallel to $\boldsymbol{p}$ and varies along the transverse direction. Such a vector potential is provided by a magnetic flux penetrating the loop hole $A_{h}$, much like in standard Aharonov-Bohm rings.

Adding to the standard quantum ring geometry, our new devices require the velocity $\boldsymbol{v}(\boldsymbol{r})$ to differ on the two paths of the interferometer. To fulfill this consideration, we arrive at the second device proposal, shown in panel (b) of Fig. 8 in comparison to the Rashba device displayed in panel (a). In contrast to the Rashba-based devices, these devices require the application of a magnetic flux penetrating $A_{h}$, but need neither spin polarization nor the Rashba effect. If we used gate potentials, they could even be made of 
Fig. 6 Suggested band structures of materials I and II. The bottom of the band of material I is offset by an energy of $V_{\mathrm{I}}$ with respect to the bottom of the band of material II. As a result, the electrons of material I have a lower velocity at the chemical potential than those of material II

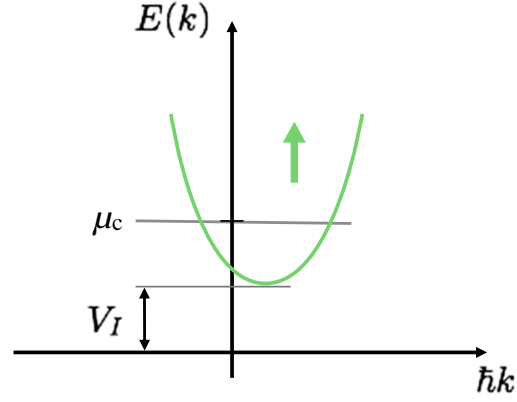

material I

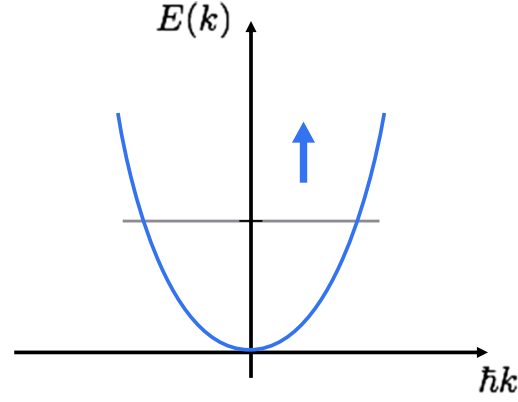

material II just one material (panel c) which would enhance interface transparency. These devices phase the same requirements on inelastic scattering as the Rashba-type device.

\section{Device Properties}

\subsection{Operation in Non-equilibrium}

We now consider the case that individual electrons are injected with a high velocity $v$ into the device. The electron energy well exceeds the temperature of the barrier $m v^{2} / 2 \gg$ $k T_{\mathrm{b}}$, corresponding to $T_{\text {con }} \gg T_{\mathrm{b}}$ in case the electron energy results from the thermal energy of the contact. If the inelastic scattering length of these high-energy electrons is comparable to the device size, these devices work according to our analysis as valves for electrons and may therefore be of interest for electronic applications, for example, to prevent feedback from output to input gates. The bandpass properties of the devices can be tuned to match the desired particle velocities by tailoring the device design such as the number of paths to the requirements. Our analysis suggests that in this operation regime the device causes an output current $\left\langle I_{\mathrm{o}}\left(B_{\mathrm{a}}\right)\right\rangle_{t}>0$ to flow in an external conductor that closes the circuit as illustrated in Fig. 9b. The devices can be combined into coherent or non-coherent circuits. The devices can be fast, because their speed is limited by the

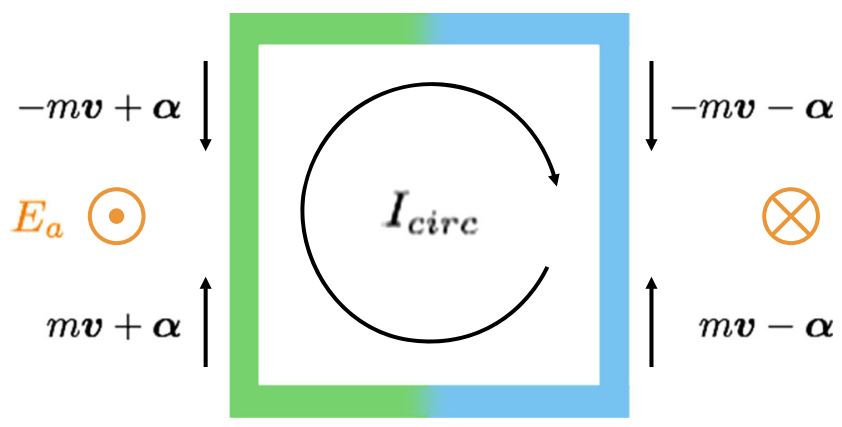

Fig. 7 Sketch of a ring consisting of two Rashba materials connected in antiparallel direction. In its ground state, the ring induces a circulating current $I_{\text {circ }}$ electron transfer time. They also can be small because they may consist of single molecules.

\subsection{Operation in Thermal Equilibrium}

These devices seem to be non-reciprocal and act as directional filters that let electrons pass in one or the other direction depending on the electron velocity. They are therefore reminiscent of Maxwellian demons. For demon action, the filter would need to operate at the temperature of the reservoirs that create the thermal electrons $\left(T_{\text {con }}=\right.$ $T_{\mathrm{b}}$ ); see Fig. 1. However, thermal scattering at the aperture that defines the electron beam and at the filter's junctions may suppress electron interference. Filters consisting of loops provided by one-dimensional conductors or suitable molecules are robust to this problem; see Fig. 9. Note that the two arms of the loops, possibly including even the beam splitters, may also be provided by an inhomogeneous tunnel barrier. For device operation, a finite phase-coherent, ballistic current of thermal electrons must be maintained across the length of the barrier $l_{\mathrm{b}}$, including the splitters, Fig. 9. In this respect, it is encouraging to note that pronounced Aharonov-Bohm oscillations of $30 \Omega$ gold rings have been measured at $\sim 0.7 \mathrm{~K}$ with ac currents of $I_{r m s}=0.2 \mu \mathrm{A}$ i.e., with electron energies $\leq 1 \mathrm{~K}$ [37]. If this tendency towards ordering prevails also for $T_{\mathrm{con}}=T_{\mathrm{b}}$ and without bias voltage is to be answered. In thermodynamic equilibrium, further effects counteract device operation because then the electrons that enter the device and the electrons that there already exist share the same thermal energy distribution. In response to the phase shifts induced, e.g., by the Rashba effect or by the Aharonov-Bohm effect, these carriers form circulating currents. These electrons affect the filtering effect on the one hand by their magnetic field and on the other hand by acting as an electron scatterer that is, however, constantly moving in one direction. We therefore cannot exclude that also in thermal equilibrium the velocity distribution of the electrons leaving the ring has a directional asymmetry.

The second law of thermodynamics demands that the open source voltage $\left\langle V_{\mathrm{o}}\left(B_{\mathrm{a}}, l_{\mathrm{c}}(v)\right)\right\rangle_{t}$ and the output current 
(a)

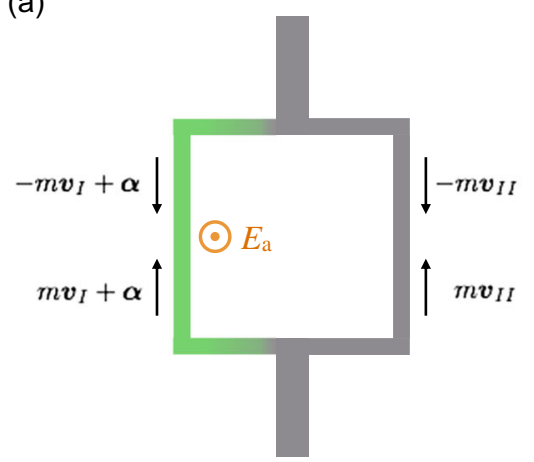

(b)

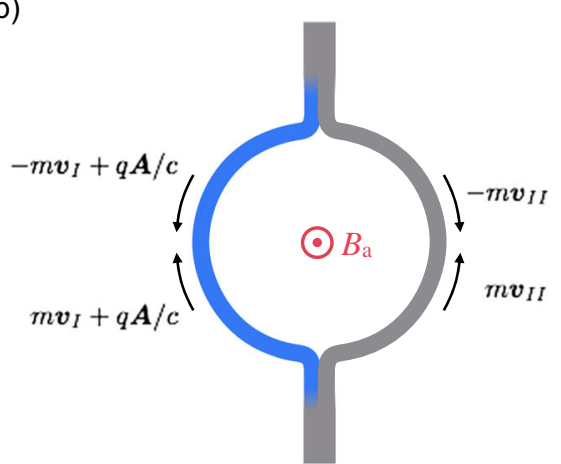

(c)

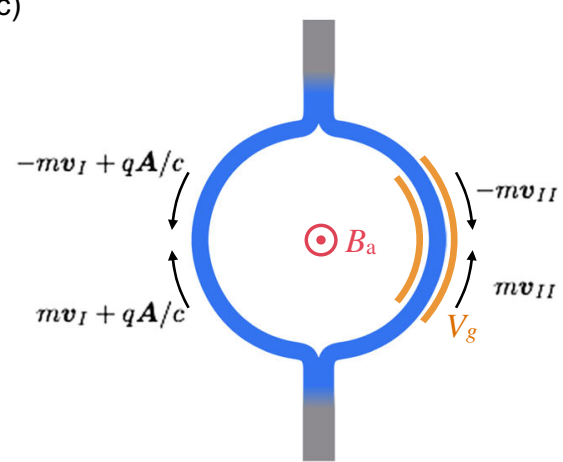

Fig. 8 Quantum rings with non-reciprocal transport based on a the Rashba effect and $\mathbf{b}, \mathbf{c}$ the Aharonov-Bohm effect. In (b), the symmetry between the electron paths I and II is broken by using two materials with different electron velocities $v$, in (c) by changing the electron

$I_{\mathrm{O}}$ flowing through an attached wire strictly disappear under all circumstances, for example for any applied magnetic field and for any mean electron coherence length $\left\langle I_{\mathrm{o}}\left(B_{\mathrm{a}}, l_{\mathrm{c}}(v)\right)\right\rangle_{t} \equiv 0$, independent of the length's functional dependence on the electron velocity. If the output current and voltage would indeed completely disappear, which mechanisms are to suppress them to precisely zero under all conditions? velocity with a gate potential $V_{\mathrm{g}}$. The devices are characterized by an inelastic scattering length that is comparable to the circumference of the loops

\section{Discussion}

In the following, we discuss several possible problems of the devices and the modeling we have used, without claiming that our discussion is complete.

(1) In our search for the devices, we have used the concept of free electrons that move with a parabolic
Fig. 9 a Length scales of a one-dimensional conductor relevant to device operation. The loop size $l_{\mathrm{b}}$ and the length of the contacts $l_{\text {con }}$ are required to obey $l_{\text {con }}>l_{\mathrm{c}} \gtrsim l_{\mathrm{b}}$, where $l_{\mathrm{c}}$ is the coherence length of the electron. b Illustration of a onedimensional conductor (dark blue) comprising an AharonovBohm ring with inelastic scattering, a non-uniform electric potential $V_{\mathrm{e}}$, and a non-uniform vector potential $\boldsymbol{A}$. This conductor is part of a loop that is closed by a standard, dissipative conductor (light blue). According to our model, a circulating current $I_{\mathrm{o}}$ flows through this loop if $T_{\text {con }}>T_{\mathrm{b}}$ (a)

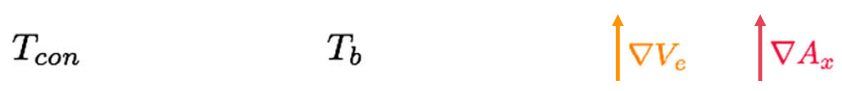

A
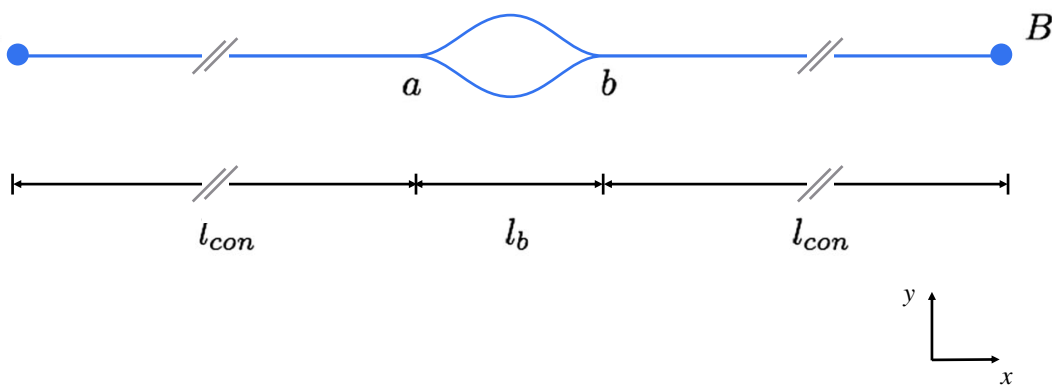

(b)

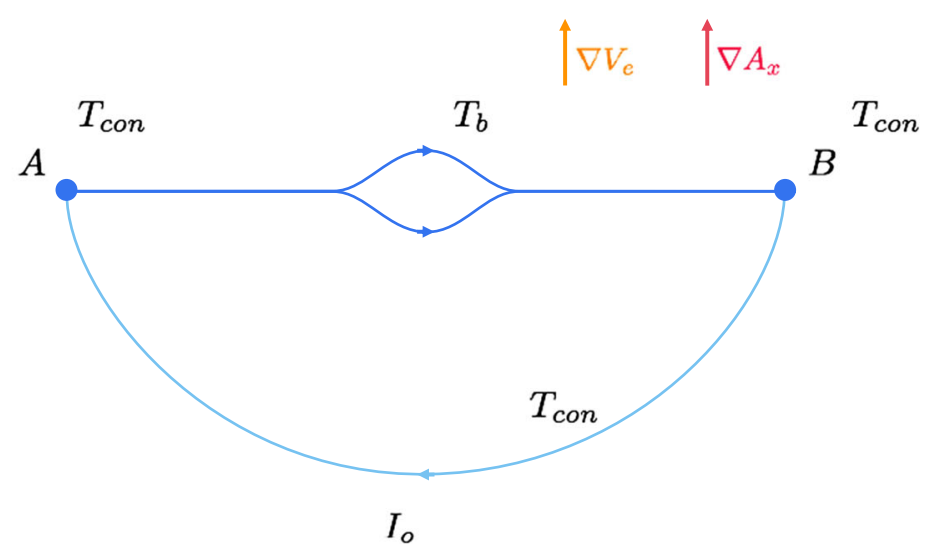


dispersion and are described by the model based on the Schrödinger equation commonly used to describe Aharonov-Bohm rings and double-slit experiments. We have also made several widely used approximations as described. The calculations, arguments, and approximations analogous to the ones performed here are based on those commonly used in the literature to describe the behavior of quantum rings and double-slit experiments.

(2) A functioning device requires that at least some coherence be maintained across the entire device. The scattering lengths have to be comparable to the device size. In this case, all contacts and wave splitters, which may be abrupt or gradual, deserve special attention. They must be transparent, i.e., they must feature sufficiently matching states for electrons passing the barrier close to the chemical potential. Contacts can never be fully transparent and they may induce phase retardation effects, which impairs device performance. Numerous observations of AharonovBohm oscillations of quantum rings operating at finite temperatures provide ample evidence that the incoherency of the contacts and at the device junctions does not necessarily inhibit device operations at finite temperatures.

(3) In devices based on the Rashba effect, electrons move in transverse magnetic fields. They are therefore subject to the Lorentz force, such that their trajectories may change or Hall fields may be generated. We have therefore limited the discussion above to the motion of electrons in 1D- or 2D-quantum wells oriented parallel to the magnetic field. Furthermore, the device proposal applies independently of whether the particles are charged or neutral. Therefore, it does not appear in principle that these effects would prevent the functioning of Rashba-based devices. In devices based on the Rashba effect, the electron phase also changes due to vector-potential contributions. The magnetic self-field of the transport current causes a magnetic flux to penetrate the hole of the device in the case where the currents of paths I and II are not the same. As discussed, such flux or stray fields induce phase shifts analogous to the Aharonov-Bohm [34] and the Aharonov-Casher [38] effects. These effects scale with the area of the loop hole, $A_{\mathrm{h}}$. However, as $A_{\mathrm{h}}$ is not a relevant parameter for the operation of the proposed device and may effectively vanish, it seems that Aharonov-Bohm-type phase changes cannot impede the performance of the Rashba-based devices in principle.

\section{Summary and Conclusions}

To achieve non-superconducting, loss-free charge transport, we suggest searching for systems with broken space and time-inversion symmetry that are subject neither to Bloch's (second) theorem nor to the reciprocity theorem. The candidates we propose are non-reciprocal devices such as the ones presented in Fig. 8. The device principles apply to all quasiparticles and particles with an electric charge or a magnetic moment.

To implement the devices, conducting asymmetric molecules, consisting for example of asymmetric rings contacted by backbone chains, are appealing candidates because of their ease of fabrication, small size, strong phase coherence (which may allow for high operation temperatures), and possibly well transparent contacts between the paths. The sorting may also be performed by using different paths in a crystal, utilizing for example transport in different bands or along different crystal directions. Topological edge and surface states are candidates of interest, too.

Based on several explicit and implicit assumptions our discussion suggests that the proposed devices show, as intended, a higher transparency for particles moving with a velocity $v$ in one direction than in the reverse, not following the reciprocity theorem, (1). Using the existing motion of the particles, the ground state of such devices exerts a sorting function on incoming particles without an electric bias or moving device components.

In our view, the concept of a state that exerts a dynamic function, for example, by means of a circulating current, deserves to be explored from a general perspective in its own right. Acting like a unidirectional membrane, like a catalyst for ordering, such a state may sort a variety of degrees of freedom, such as spins, orbital momentum, valley occupancy, and velocity, and may use mechanisms other than quantum interference. Corresponding devices are therefore driven by quantum mechanical phenomena, but, if incoherent scattering is involved, operate at the interface between quantum physics and classical physics.

For $T_{\text {con }}>T_{\mathrm{b}}$, we foresee that the proposed devices induce finite currents in normal, dissipative conductors. For $T_{\text {con }}=T_{\mathrm{b}}$, the second law of thermodynamics requires that the time-averaged output voltages and currents of such a device cancel precisely for any ring designs and contact configuration, at any applied magnetic field and for any coherence length: $\left\langle V_{\mathrm{o}}\left(B_{\mathrm{a}}, l_{\mathrm{c}}(v)\right)\right\rangle_{t} \equiv 0,\left\langle I_{\mathrm{o}}\left(B_{\mathrm{a}}, l_{\mathrm{c}}(v)\right)\right\rangle_{t} \equiv$ 0 . Because the proposed ground states have a tendency to enhance order in attached electron reservoirs, it is a question to us whether the output current and voltage indeed strictly disappear for $T_{\mathrm{con}}=T_{\mathrm{b}}$, and if so, by which 
microscopic mechanisms. These devices therefore permit novel and exacting tests of the second law.

Acknowledgements Open access funding provided by Max Planck Society. We gratefully acknowledge an outstanding interaction with D. Braak and T. Kopp, very helpful discussions with or support by them, A. Alavi, A. Brataas, A.V. Boris, H. Boschker, E. Fillis-Tsirakis, P. Bredol, S. Hellberg, P. Horsch, G. Khaliullin, V. Kresin, L. Kürten, J. Maier, D. Manske, L. Pavka, M. Randeria, C. Schön, H. Takagi, R. Valenti, D. van Harlingen, and R. Wanke. I am grateful to T. Kopp for pointing to the equivalency of $\boldsymbol{A}(\mathbf{r})$ and $\boldsymbol{\alpha}(\mathbf{r})$ in (10).

Open Access This article is distributed under the terms of the Creative Commons Attribution 4.0 International License (http://creativecommons. org/licenses/by/4.0/), which permits unrestricted use, distribution, and reproduction in any medium, provided you give appropriate credit to the original author(s) and the source, provide a link to the Creative Commons license, and indicate if changes were made.

\section{References}

1. Kamerlingh Onnes, H.: Commun. Phys. Lab. Univ. Leiden 120b (1911), reprinted in Proc. K. Ned. Akad. Wet. 13, 1274 (1911)

2. Bednorz, J.G., Müller, K.A.: Z. Phys. B 64, 189 (1986)

3. Drozdov, A.P., Eremets, M.I., Troyan, I.A., Ksenofontov, V., Shylin, S.I.: Nature 525, 73 (2015)

4. Bloch, F.: Phys. Rev. 137, A787 (1965)

5. Büttiker, M., Imry, Y., Landauer, R.: Phys. Lett. 96A, 365 (1983)

6. Lévy, L.P., Dolan, G., Dunsmuir, J., Bouchiat, H.: Phys. Rev. Lett. 17, 2074 (1990)

7. v. Klitzing, K., Dorda, G., Pepper, M.: Phys. Rev. Lett. 45, 494 (1980)

8. Weis, J., v. Klitzing, K.: Phil. Trans. R. Soc. A 369, 3954 (2011)

9. Qi, X.-L., Zhang, S.-C.: Rev. Mod. Phys. 83, 1057 (2011)

10. Zhang, X., Zhang, S.-C.: Proc. SPIE 8373, 837309 (2012)

11. Feynman, R.P., Leighton, R.B., Sands, M.: The Feynman Lectures on Physics, vol. I. Addison-Wesley, Boston (1964)

12. Astumian, R.D., Hänggi, P.: Phys. Today, November 2002, 33
13. Maxwell, J.C.: Theory of Heat. Longmans Green, and Co., London (1871)

14. Bohm, D.: Phys. Rev. 75, 502 (1949)

15. Ohashi, Y., Momoi, T.: J. Phys. Soc. Jpn. 65, 3254 (1996)

16. Landau, L.D., Lifshitz, E.M.: Quantum Mechanics: nonRelativistic Theory, vol. 3 of Course of Theoretical Physics, 2nd edn. Pergamon Press, Oxford (1959)

17. Bilhorn, D.E., Foldy, L.L., Thaler, R.M., Tobocman, W.: J. Math. Phys. 5, 435 (1964)

18. Deák, L., Fülöp, T.: Ann. Phys. 327, 1050 (2012)

19. Kristić, V., Roth, S., Burghard, M., Kern, K., Rikken, G.L.J.A.: J. Chem. Phys. 117, 11315 (2002)

20. Wakatsuki, R., Saito, Y., Hoshino, S., Itahashi, Y.M., Ideue, T., Ezawa, M., Iwasa, Y., Nagaosa, N.: Sci. Adv. 3, e1602390 (2017)

21. Adam, J.D., Davis, L.E., Dionne, G.F., Schloemann, E.F., Stitzer, S.N.: IEEE Trans. Microw. Theory Tech. 50, 721 (2002)

22. Potton, R.J.: Rep. Prog. Phys. 67, 717 (2004)

23. Rashba, É.I.: Sov. Phys. Solid State 2, 1109 (1960)

24. Manchon, A., Koo, H.C., Nitta, J., Frolov, S.M., Duine, R.A.: Nat. Mater. 14, 871 (2015)

25. Volkov, B.A., Kopaev, Yu.V.: Pis'ma Zh. Eksp. Teor. Fiz. 27, 10 (1978). [JETP Lett. 27, 7 (1978)]

26. Eliashberg, G.M.: Pis'ma Zh. Eksp. Teor. Fiz. 38, 188 (1983). [JETP Lett. 38, 220 (1983)]

27. Tavger, B.A.: Phys. Lett. A 116, 123 (1986)

28. Blount, E.I.: Phys. Rev. 38, 6711 (1988)

29. Vedyayev, A., Ryzhanova, N., Strelkov, N., Dieny, B.: Phys. Rev. Lett. 110, 247204 (2013)

30. Zhuravlev, M.Y., Vedyayev, A.V., Titova, M.S., Ryzhanova, N.V., Gusakova, D.: J. Magn. Magn. Mater. 441, 572 (2017)

31. Ast, C.R., Henk, J., Ernst, A., Moreschini, L., Falub, M.C., Pacile, D., Bruno, P., Kern, K., Grioni, M.: Phys. Rev. Lett. 98, 186807 (2007)

32. Frustaglia, D., Richter, K.: Phys. Rev. B 69, 235310 (2004)

33. Tserkovnyak, Y., Brataas, A.: Phys. Rev. B 76, 155326 (2007)

34. Aharonov, Y., Bohm, D.: Phys. Rev. 115, 485 (1959)

35. Landauer, R., Büttiker, M.: Phys. Rev. Lett. 54, 2049 (1985)

36. Imry, Y.: Introduction to Mesoscopic Physics. Oxford University Press, Oxford (2002)

37. Webb, R.A., Washburn, S., Umbach, C., Laibowitz, R.B.: Phys. Rev. Lett. 54, 2696 (1985)

38. Aharonov, Y., Casher, A.: Phys. Rev. Lett. 53, 319 (1984) 